\title{
Hybrid method for automating generation of reticulated structures (lattice structures) in complex conception domains
}

\author{
Zineb Biallaten, Raddouane Chiheb, Abdellatif El Afia \\ National School of Computer Science and Systems Analysis (ENSIAS), Mohamed V University, Morocco
}

\begin{tabular}{|c|c|}
\hline Article Info & ABSTRACT \\
\hline Article history: & \multirow{9}{*}{$\begin{array}{l}\text { A reticulated structure is an interconnexion of bars used to create industrial } \\
\text { products. They are rigid and lighter than traditional structures. So they can be } \\
\text { the best choice when material gain is an optimization purpose. Generating a } \\
\text { reticulated structure automatically is a feature helping industrial players in } \\
\text { the design phase. This generation depends on the kind of the conception } \\
\text { domain. In this paper we propose a solution that generates a reticulated } \\
\text { structure in an arbitrary domain with zero or several holes. The proposed } \\
\text { solution is a hybrid method using a technique generating a reticulated } \\
\text { structure in a convex conception domain and suggesting a criterion to } \\
\text { validate generated segments. Our new algorithm uses a method of } \\
\text { computational geometry. We also present a study of the behaviour of a } \\
\text { reticulated structure generated using our tool by calculating the deformation } \\
\text { energy of this structure. }\end{array}$} \\
\hline Received May 16, 2018 & \\
\hline Revised Sept 30, 2018 & \\
\hline Accepted Oct 19, 2018 & \\
\hline Keywords: & \\
\hline Deformation energy & \\
\hline Lattice structure & \\
\hline Point-in-polygon & \\
\hline Reticulated structure & \\
\hline
\end{tabular}

Copyright () 2019 Institute of Advanced Engineering and Science. All rights reserved.

\section{Corresponding Author:}

Zineb Biallaten,

National School of Computer Science and Systems Analysis (ENSIAS),

Mohamed V University,

Avenue Mohammed Ben Abdallah Regragui, Madinat Al Irfane, BP 713, Agdal, Rabat, Morocco.

Email: zineb.biallaten@um5s.net.ma

\section{INTRODUCTION}

Automating the design process of a structure is an industrial challenge. That's why design tools are continually improving to invent products with optimum structures while simulating their behavior during the design phase. In this context, reticulated structures known for their rigidity and low weight seem to be the best response to a need for optimal structure with minimal cost.

A reticulated structure is a union of $\mathrm{N}$ cylindrical rods interconnected at their extremities. They are light structures. This property coincides with the industry's need to save the raw material by designing lightweight components, because weight reduction also reduces the cost of the component. These structures have also the advantage of being prefabricated. They are present in civil engineering, mechanic, architecture, etc. For example, they are used to build bridges, aircrafts.

In general, a structure used in industry must be in optimal form otherwise it could be inappropriate for production. So, structure optimization methods are usually conscripted to find a better distribution of the material in designed structure. By studying some of these methods, we notice that a good part of the proposed solutions can be considered as reticulated structure. That is why, we suggest designing a structure as a reticulated structure from the beginning.

Desiging a reticulated structure is doing in two phases. First, the structure is generated automatically inside a conception domain choosen by the designer. Then the generated structure undergoes optimization treatment to obtain the best possible structure.

In this paper we propose a method for the automatic generation of a reticulated structure. Designing a reticulated structure depends on the conception domain and especially on its convexity. In an industrial context, conception domains may have any form. It was therefore necessary to study the possibility of 
generating a reticulated structure whatever the nature of the initial design domain and especially if it is not convex.

On the one hand, the generation of a reticulated structure amounts to creating bars interconnected at the ends. On the other hand, by defining a design domain, the designer determines the envelope where the structure is to be set up. Thus, it determines the limits of the product in space. Now, if this domain is not convex then a generated bar may not be contained completely in the design domain and therefore it must be eliminated.
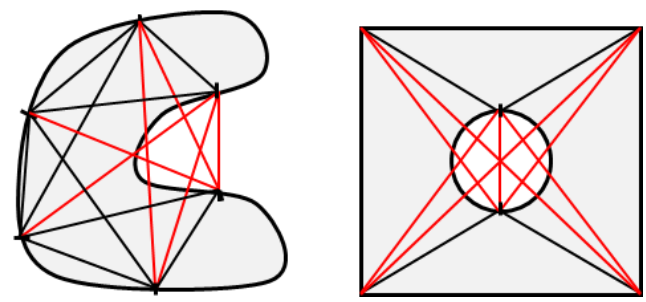

Figure 1. Generating a reticulated structure for a non convex conception domain. (Segments to be eliminated are red) (a) Conception domain without holes; (b) Conception domain with a hole

Thus, the automatic generation of a reticulated structure begins by linking the points discretizing the boundary of the design domain as showing in Figure 1. Then, the bars that are not entirely contained in the design area will be eliminated. And finally, the bars retained will be redistributed to increase the rigidity of the structure. However finding a bar removal criterion is not so obvious.

In this paper, we suggest an algorithm to generate a reticulated structure automatically whatever the type of the design domain. Our new algorithm is defined in two main phases. It starts by connecting all initial nodes of the structure. Then, it proposes an elimination criterion to reject segments that are not included entirely in the conception domain. This elimination is based on a method of computational geometry.

The paper is divided into five sections. Section 2 situate the problem by introducing the basics on the generation of reticulated structures, and gives a brief overview of previous works done on this subject. The new algorithm is explained in Section 3. In Section 4, an overview of the structure's deformation energy is given to introduce the optimization work of the generated structure. Finally, in Section 5, conclusions are drawn.

\section{PROBLEM SITUATION}

\subsection{Generating a reticulated structure}

The algorithms for generating reticulated structures differ according to the type of the design domain. In a convex domain $\Omega$, two points A and B are connected by a segment contained entirely in $\Omega$. This property has been exploited by Chiheb\&Panasenko [1] to generate automatically reticulated structures from a convex design domain. Their technique, named AGRS, begins with a discretization of the boundary of the design domain into a finite number of points. Then, these points are linked in pairs to represent the reticulated structure which is then subjected to an optimization treatment enabling to obtain a most optimal structure. Figure 2 presents an example of the reticulated structure generation using AGRS.

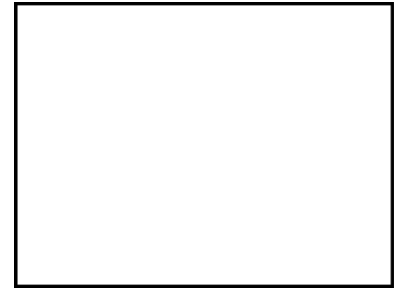

(a)

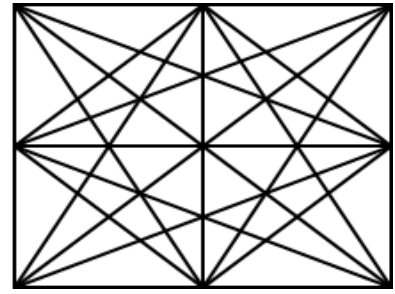

(b)

Figure 2. Generating a reticulated structure in a convex domain (a) Convex conception domain;

(b) Corresponding reticulated structure 
Therefore, a convex design domain is a special case of design domains, and all domains are not convex. By studying non-convex design domains, we noticed that there are non-convex domains that may be decomposed into a finite number of convex sub-domains. So, we recently proposed an algorithm [2] to generate and optimize a reticulated structure from a design domain of this category. The proposed algorithm uses a convex decomposition technique to obtain a finite number of convex sub-domains. Each of these subdomains is considered to be an input of the Automatic Generation of Reticulated Structures algorithm (AGRS) [1]. Therefore, a reticulated structure is generated by a convex sub-domain. Subsequently, the partial solutions are merged to obtain the overall reticulated structure. All these steps were presented in Figure 3.
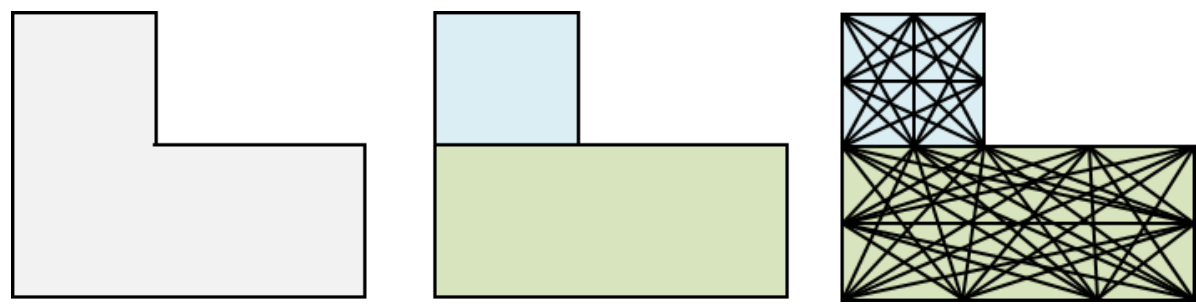

Figure 3. Decomposing a non-convex domain and Generating a reticulated structure

A second category of non-convex design domains is those that cannot be decomposed into exactly convex sub-domains. The complexity of these areas increases if they contain holes. We are interested in this type of non-convex domains.

\section{PROPOSED SOLUTION}

The automatic generation of a reticulated structure begins with a discretization of the contour $\partial \Omega$ into a finite number of points. Each point of discretization is a node of the structure. Then, the nodes are linked in pairs to create the segments of the reticulated structure. The set of created segments is called E. Now a segment is eliminated if it is not completely contained in the design domain, in other words:

Let $\Omega$ be the non-convex design domain, the segment $S$ must be eliminated if:

$$
\dot{\hat{S}} \cap\left(\mathbb{R}^{2} \backslash \Omega\right) \neq \varnothing
$$

The set of segments to be eliminated is defined as follows:

$$
\mathrm{ESAE}=\left\{S / \dot{\hat{S}} \cap\left(\mathbb{R}^{2} \backslash \Omega\right) \neq \emptyset\right\}
$$

The process of automatic generation is illustrated in Figure 4. According to (5), a segment $\mathrm{S}$ is eliminated if there is a point of its interior which does not belong to $\Omega$.

In Figure 5, the two segments $S_{A B}$ and $S_{B C}$ are to be eliminated. For $S_{B C}$, all points of its interior do not belong to the design domain. So a single test is enough to eliminate this type of segment. However, the segment $S_{A B}$ is partially contained in $\Omega$ so finding a point that does not belong to $\Omega$ can be expensive since it may require a large number of tests.

Let $S_{p_{i} p_{j}}$ be a segment of the structure, and $\mathrm{M}\left(\begin{array}{l}\mathrm{x} \\ \mathrm{y}\end{array}\right)$ a point of $S_{p_{i} p_{j}}$ :

if M does not belong to $\Omega$ then $S_{p_{i} p_{j}}$ is eliminated automatically.

First, it was important to set up a technique to choose the points of the segment for which the relation (6) must be verified. Since a segment is infinity of points, we were inspired by a bracketing method to represent the segment with a set of points $M\left(\begin{array}{l}x \\ y\end{array}\right)$. The bracketing is a method used to decide on the existence of zeros of a given function in an interval. This method proposes to subdivide an interval into a finite number of sub-intervals with the same size, and then the research process is applied to each subinterval.

But, determining whether a point belongs to a domain is not a simple problematic. Therefore, we propose to use a geometry algorithm to solve this problem. It's an algorithm that is able to determine the

Hybrid method for automating generation of reticulated structures (lattice structures)... (Zineb Biallaten) 
position of a point with respect to a polygon: inside, outside or on the edges of the polygon. For our case, the polygon is the approximation of the initial design domain created by linking the discretization nodes of the boundaries of the design domain (including the boundaries of the holes).

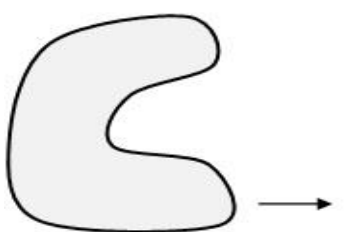

(a)

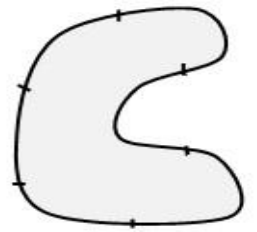

(b)

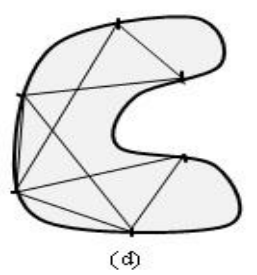

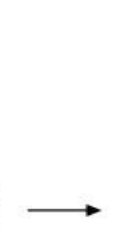

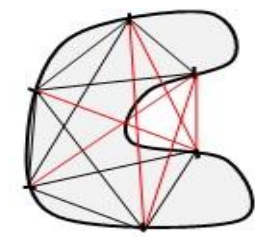

(c)

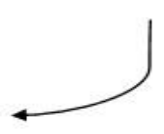

Figure 4. Generating a reticulated structure steps (a) Non-convex
conception domain; (b) Discretization; (c) Creating segments; conception domain; (b) Discretization; (c) Creating
(d) Eliminating improper segments

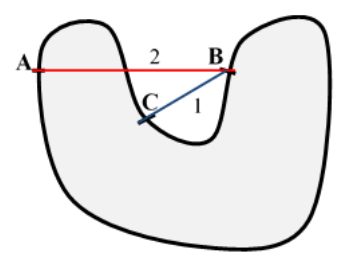

Figure 4. Segments to eliminate

\subsection{Point in polygon: review}

The point in polygon is a very natural problem in the field of computational geometry which decidewhether a point is inside or outside a polygon. So, we want to use this technique to check each point of a studied segment and decide whether it is inside the studied conception domain.

Many algorithms are used in literature to locate a point relative to a polygon. Two examples are shown on Figure 6. One of the first point-in-polygon algorithms is Crossing Number algorithm (CNA) [3]-[5]. Given a polygon $\mathrm{P}$ and a point $\mathrm{M}, \mathrm{CAN}$ counts the number of times a ray starting from the point $\mathrm{M}$ crosses the polygon boundary edges. The point is outside when this "crossing number" is even; otherwise, when it is odd, the point is inside. This method is efficient for all types of polygons and it works in $O(n)$ time ( $\mathrm{n}$ is the number of the polygon edges) [6].
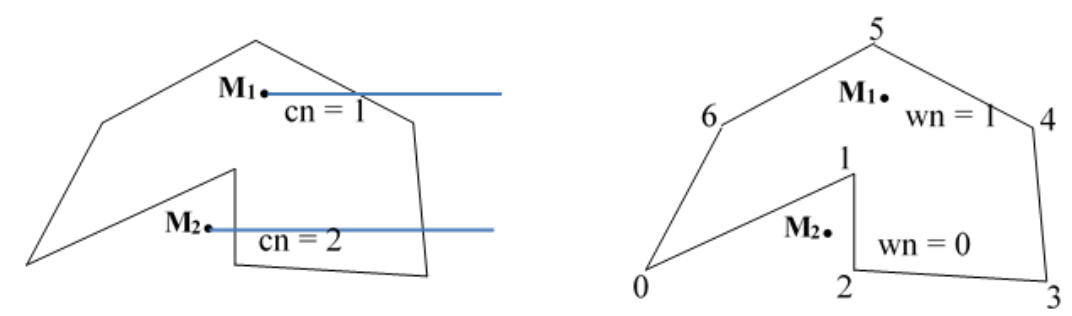

Figure 5. (a) Crossing number method; (b) Winding number method

For the winding number algorithm (WNA) [5], it counts the number of times the polygon winds around the point $\mathrm{M}$. The point is outside only when this "winding number" is zero; otherwise, the point is inside. This algorithm is not desirable for polygons with a large number of sets. However, an improved implementation of the winding number was proposed by Hormann\&Agathos [7] for self-intersecting polygons.

WNA \& CAN are algorithms based on computing a parameter. They are simple to implement but both of them are slow especially if the number of polygon's vertices is large. So, other algorithms were proposed to improve the performance of solutions. These algorithms adopt the principle of "divide and 
conquer". So, they propose a pre-processing step dividing the polygon into a set of simpler polygons (convex polygons [8], triangles [9], and trapezoids [10]). After decomposition each algorithm uses one of the basic algorithms to determine the position of the point in question.

\subsection{New algorithm steps}

The solution that we propose in this work is a hybrid algorithm using the principles of the AGRS algorithm [1] to generate a reticulated structure in an arbitrary conception domain. The steps of this new algorithm are as follows:

Step 1: The designer gives the design domain. It is enough that he defines the outer border of this domain and possibly the borders of the holes (if they exist).

Step 2: $\quad$ Borders are discretized with a sufficient number of points. These points are the initial nodes of the future structure.

Step 3: All points are linked in pairs. Each link represents a candidate segment of the reticulated structure. Some segments are not completely contained in the conception domain. So, they are eliminated in step 4.

Step 4: For each segment $S_{p_{1} p_{2}}\left(P_{1}\left(\begin{array}{l}x_{1} \\ y_{1}\end{array}\right) \& P_{2}\left(\begin{array}{l}x_{2} \\ y_{2}\end{array}\right)\right.$ are two points of step 2), we apply a point-in-polygon algorithm to maintain or eliminate this segment. However, applying this type of algorithms on the design domain assumes that it is a closed polygon. So, the boundary near the segment must be approximated to a closed polygon.

$S_{p_{1} p_{2}}$ is discretized into a set of points $M\left(\begin{array}{l}x \\ y\end{array}\right)$. So, checking the elimination criterion for the segment is to check if each $M\left(\begin{array}{l}x \\ y\end{array}\right)$ is inside or outside the "polygon":

For each $M\left(\begin{array}{l}x \\ y\end{array}\right)$, Call an algorithm Point- In- Polygon,

toEliminate $=$ PointInPolygon $(M)$

if toEliminate $=$ true then $S_{p_{1} p_{2}}$ is to eliminate

Step 5: After eliminating inappropriate segments, we determine the points of intersection between the validated segments. Then, we calculate the connectivity matrix of the generated structure.

Figure 7 shows an example of implementation of our proposed algorithm.
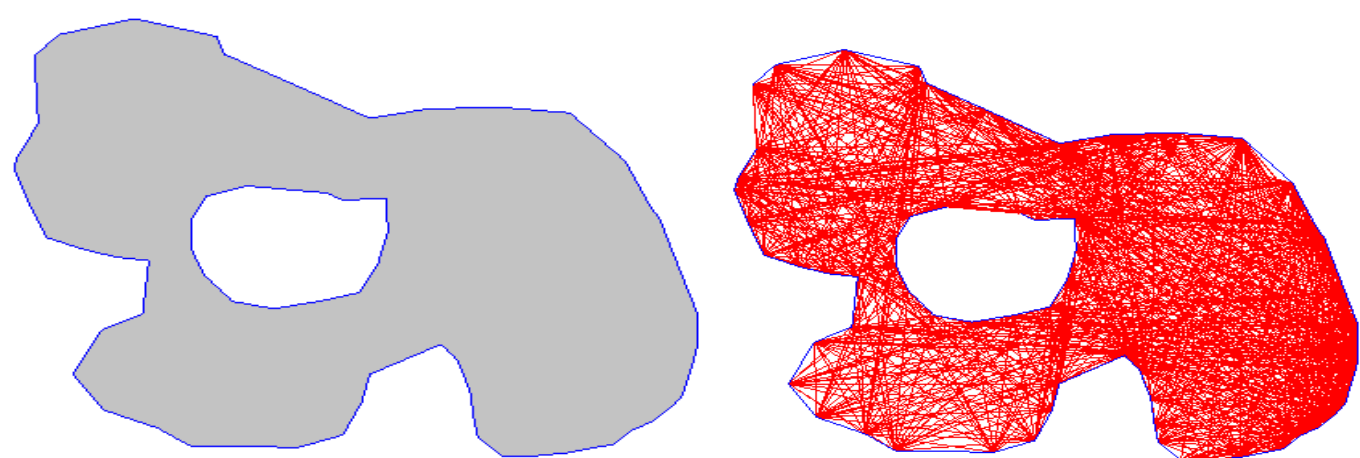

Figure 6. Automatic generation of a reticulated structure in an arbitrary non-convex conception domain

The solution we propose is hybridization between two solutions. First we take advantage of the simplicity of the principle of generating reticulated structures in a convex domain. Then, we validate each segment using a reduced complexity algorithm.

Moreover, if the number of discretization points is large then the number of segments to test is important. So it was interesting to design our solution as a parallel program. Parallelism reduces the running time and optimizes the use of material resources which increase the efficiency of our algorithm. This mechanism could be used in two contexts:

a. To select the segment to be processed: Segments can be shared between multiple processes

b. Sharing the tests of the elimination criterion between several processes. So each segment would be treated by many processes

Our method is based on discretizing the studied segment. So, it is sensitive to the number of the discretization points. In some cases, this number should be sufficient to detect the points that are out of the conception domain.

Hybrid method for automating generation of reticulated structures (lattice structures)... (Zineb Biallaten) 
In Figure 8, the conception domain has a reflex vertex with an angle that tends to $2 \pi$. As shown on the figure, the discretization of the segment must create a sufficient number of points on the diameter of the circle to decide that the segment must be eliminated. The experiments have shown that if the bridge created by such an angle is of the order of $10^{-6}$, a number of 200 points is sufficient to eliminate the segment see Figure 9.

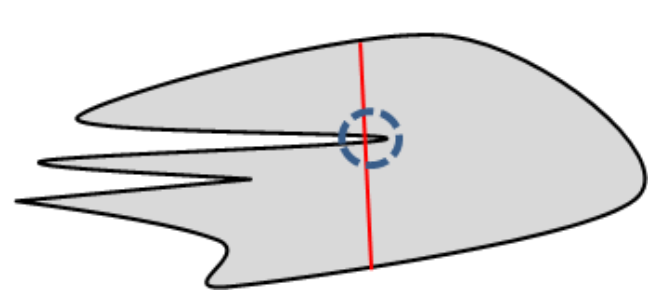

Figure 7. Special case

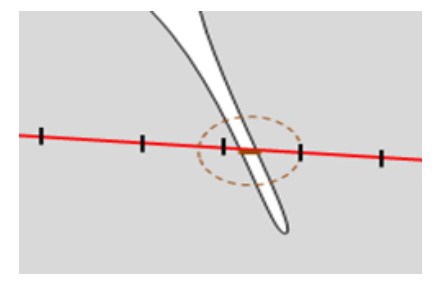

Figure 8 . Bridge of order of $10^{-6}$

Until now, the designer has to choose the step of discretization. But, it would be interesting to automate this step according to the nature of the conception domain.In the other hand, the method we use to validate a segment has for inputs a point and a polygon. But our design domains are not necessarily polygons but arbitrary curves. Then, it was necessary to approximate the contours of the design domain to a polygon such that its sides are the segments linking two points $P_{i}$ and $P_{i+1}$. This approximation should be concise to guarantee that it would not impact the results. So, we choose a discretization fine enough to approach the continuity of the design domain.

\section{NODAL DISPLACEMENT}

A reticulated structure is designed to be rigid and balanced. Moreover it can be subjected to mechanical or thermal forces of deformation influencing its stiffness. These forces are applied on the nodes of the structure what produces a displacement of these nodes.

Optimizing a reticulated structure means minimizing its deformation energy. This energy is defined using the nodal displacement matrix:

$$
E_{\text {def }}=\frac{1}{2} U^{T} F
$$

$\mathrm{U}$ : nodal displacement vector

$\mathrm{F}$ : vector of nodal forces

So, after generating the structure we need to calculate the deformation energy. We start by calculating the nodal displacements. For this, we use an equation defining a relation between stiffness matrix, vector of nodal displacements and vector of nodal forces:

$$
K U=F
$$

such that: $K$ is a square matrix of size $2 \times \mathrm{n}$ (n is the number of the structure nodes); $U$ and $F$ are vectors of size $2 \times n$.

We suggest in our tool a feature to calculate the deformation energy of the generated structure in order to introduce the optimization works. So, three inputs are to be defined: Connectivity matrix, nodes with zero displacement and the vector of nodal forces.

The generation operation, presented in this paper, produces a reticulated structure represented by a connectivity matrix. A connectivity matrix is produced to be used for describing the equilibrium equation of this structure. It is a square binary matrix that describes connections between the nodes of the structure. A connection between two points is equivalent to a segment. Also, for any structure, we have nodes with zero displacement and nodes that are under load. Those that are zero displacement must be neglected when calculating nodal displacements.

Experiments have shown that as the transverse section grows larger the deformation energy decreases see Figure 10. However, to choose the best transverse distribution it may be useful to use bars with different transverse sections. 
Also, the material to be used to make the bars of the reticulated structure has a significant impact on the deformation energy see Figure 11. This is due to the fact that the deformation energy is calculated using a constant named Young Module. This constant differs according to the material used for the manufacture of the bars.

It is important to simulate thebehavior of the structure to study its stability and its rigidity.But this simulation is not enough and we must also make sure to design it in the best model. For our project, we defined more than a level of optimization and we aim to use a metaheurstic algorithm to reach our goal. A metaheuristic algorithm solves hard problems for which we can not have exact solutions with classical methods.In literature, several algorithms were proposed to define an optimal structure [12], [13] and a set of algorithms was destined to optimize lattice structures [14]-[16]. So this topic of optimization will be detailled in future work.

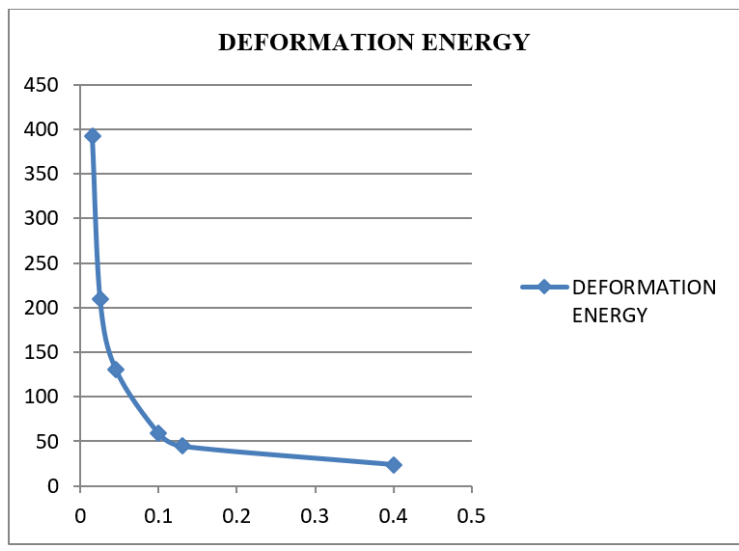

Figure 9. Variation of the deformation energy depending on the transverse section of the bars

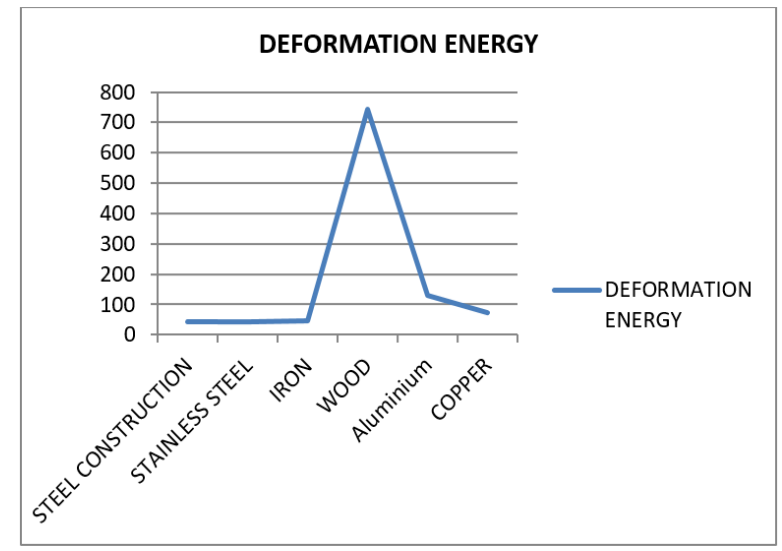

Figure 10. Impact of the manufacturing material of the bars on the energy of deformation

\section{CONCLUSION}

This work aims to create a tool helping industrial engineers to design reticulated structures in efficient way. The designer only gives the envelope of the structure and our tool takes care of the generation of the structure with an optimum form. In this paper, we developed an algorithm to generate reticulated structures in arbitrary conception domain. The algorithm is a generalization of algorithms generating reticulated structures for special cases of conception domains. This solution is working on complex conception domains even those with holes.

A tool for industrial design must study the behavior of the structure to be designed. The study of this behavior would lead us to choose the selection of the bars constituting the optimal form of the structure. Now, optimizing the structure amounts to propose the form that minimizes its deformation energy. So, we present here an example of generated structure and the calculation scheme of this energy, starting with a calculation of the nodal displacements of the structure.

\section{REFERENCES}

[1] Chiheb, R. and Panasenko, G.,"A Novel Algorithm for the Conception and Optimization of Reticulate Structures Applicable Analysis," vol. 91(5), pp. 1019-1027, 2012.

[2] Biallaten, Z., Chiheb, R., El Afia, A., and Faizi, R.,"Novel Method to Generate and Optimize Reticulated Structures of a Non-Convex Conception Domain," International Journal of Software Engineering and Its Applications, vol. 11(2), pp 17-26, 2017.

[3] Galetzka, M., and Glauner, P.,"A Simple and Correct Even-Odd Algorithm for the Point-in-Polygon Problem for Complex Polygons," In Proceedings of the 12th International Joint Conference on Computer Vision, Imaging and Computer Graphics Theory and Applications (VISIGRAPP 2017), vol. 1: GRAPP, 2017.

[4] Haines, E.,"Pointin Polygon Strategies," Graphics gems IV, vol. 994, pp 24-26, 1994.

[5] J. D. Foley, A. van Dam, S. K. Feiner, J. F. Hughes,"Computer Graphics-Principles and Practice 2nd ed," AddisonWesley, Reading, MA, 1990.

[6] Žalik, B. and Kolingerova, I.,"A Cell-based Point-in-polygon Algorithm Suitable for Large Sets of Points," Computers \& Geosciences, vol. 27(10), pp. 1135-1145, 2001. 
[7] Hormann, K., \&Agathos, A.,"ThePoint in Polygon Problem for Arbitrary Polygons," Computational Geometry, vol. 20(3), pp. 131-144, 2001.

[8] Li, J., Wang, W.C., Wu, E.H.,"Point-in-polygon Tests by Convex Decomposition," Computers \&Graphics, vol.31 (4), pp. 636-648, 2007.

[9] Feito, F., Torres, J.C., Urena, A.,"Orientation, Simplicity, and Inclusion Test for Planar Polygons," Computers \& Graphics, vol. 19(4), pp. 595-600, 1995.

[10] Zalik, B., Clapworthy, G.J., "A Universal Trapezoidation Algorithm for Planar Polygons," Computers \& Graphics, vol.23 (3), pp. 353-363, 1999.

[11] Chiheb, R. and Panasenko, G. P."Optimization of Finite Rod Structures and L-convergenc," Journal of dynamical and control systems, vol. 4(2), pp. 273-304, 1998.

[12] Wu, S. and Su, T.,"Optimization Design of Cantilever Beam for Cantilever Crane Based on Improved GA," Indonesian Journal of Electrical Engineering and Computer Science, vol. 12(4), pp. 2652-2657, 2014.

[13] Sun, Y., Wu, Q. and Yan, X.,"An Improved Constrained Engineering Optimization Design Algorithm," Indonesian Journal of Electrical Engineering and Computer Science, vol. 12(11), pp. 7079-7978, 2014.

[14] Gonçalves, M. S., Lopez, R. H., and Miguel, L. F. F.,"SearchGroup Algorithm: a New Metaheuristic Method for the Optimization of Truss Structures," Computers \& Structures, vol. 153, pp. 165-184, 2015.

[15] Zhang, J., Luo, M., Gao, R., and He, L.,"Optimization of Actuators in Smart Truss Based on Genetic Algorithms," Indonesian Journal of Electrical Engineering and Computer Science, vol. 10(7), pp. 1615-1620, 2012.

[16] Agarwal, S., and Vasan, A.,"ComputationalStrategy for Structural Analysis, Design, and Optimization of Trusses using Genetic Algorithm and Particle Swarm Optimization," in Advanced Computing (IACC).IEEE 2016 6th International Conference onIEEE, pp. 203-207, 2016.

\section{BIOGRAPHIES OF AUTHORS}

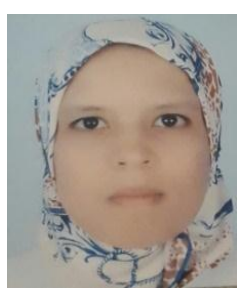

Zineb Biallaten received her engineer degree in Computer science in 2009 at the National School of computer science and systems analysis (ENSIAS) School, Rabat, Morocco. In 2015, she joined the RIIT Laboratory of Mohamed V University, Rabat, Morocco. Her actual main research interests concern Structure Optimization. E-mail: zineb.biallaten@um5s.net.ma

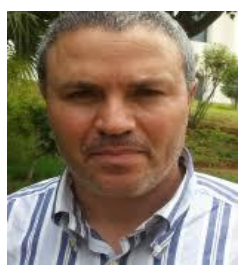

Dr. Raddouane Chiheb is a professor of applied mathematics at the National School of Computer Science and Systems Analysis at Mohammed V University, Rabat, Morocco. He obtained his Master from the National Institute of Applied Sciences of Lyon and PhD from the Jean Monnet University of Saint-Etienne. His research interests are in the area of Semantic Analysis, Structural Optimization, Education, Optimization of the logistics chain, and Value Analysis. He supervised over 10 students. Prof. RaddouaneChiheb is President of the Moroccan Association for the Value Analysis. Email: r.chiheb@um5s.net.ma

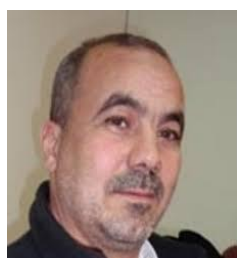

Dr. Abdellatif El Afia is an Associate Professor at National School of Computer Science and Systems Analysis (ENSIAS), Rabat, Morocco. He received his M.Sc. degrees in Applied Mathematics from University of Sherbrook. He obtained his Ph.D. in 1999 in Operation Research from University of Sherbrook, Canada. Research areas of interest are Mathematical Programming (Stochastic and deterministic), Metaheuristics, Recommendation Systems and Machine Learning. Email: a.elafia@um5s.net.ma 